\title{
Astropy: A community Python package for astronomy
}

The Astropy Collaboration, Thomas P. Robitaille ${ }^{1}$, Erik J. Tollerud ${ }^{2,3}$, Perry Greenfield ${ }^{4}$, Michael Droettboom ${ }^{4}$, Erik Bray ${ }^{4}$, Tom Aldcroft ${ }^{5}$, Matt Davis ${ }^{4}$, Adam Ginsburg ${ }^{6}$, Adrian M. Price-Whelan ${ }^{7}$, Wolfgang E. Kerzendorf ${ }^{8}$, Alexander Conley ${ }^{6}$, Neil Crighton ${ }^{1}$, Kyle Barbary ${ }^{9}$, Demitri Muna ${ }^{10}$, Henry Ferguson ${ }^{4}$, Frédéric Grollier ${ }^{12}$, Madhura M. Parikh ${ }^{11}$, Prasanth H. Nair ${ }^{12}$, Hans M. Günther ${ }^{5}$, Christoph Deil ${ }^{13}$, Julien Woillez ${ }^{14}$, Simon Conseil ${ }^{15}$, Roban Kramer ${ }^{16}$, James E. H. Turner ${ }^{17}$, Leo Singer ${ }^{18}$, Ryan Fox ${ }^{12}$, Benjamin A. Weaver ${ }^{19}$, Victor Zabalza ${ }^{13}$, Zachary I. Edwards ${ }^{20}$, K. Azalee Bostroem ${ }^{4}$, D. J. Burke ${ }^{5}$, Andrew R. Casey ${ }^{21}$, Steven M. Crawford ${ }^{22}$, Nadia Dencheva ${ }^{4}$, Justin Ely ${ }^{4}$, Tim Jenness ${ }^{23,24}$, Kathleen Labrie ${ }^{25}$, Pey Lian Lim ${ }^{4}$, Francesco Pierfederici ${ }^{4}$, Andrew Pontzen ${ }^{26,27}$, Andy Ptak $^{28}$, Brian Refsdal ${ }^{5}$, Mathieu Servillat ${ }^{29,5}$, and Ole Streicher ${ }^{30}$

1 Max-Planck-Institut für Astronomie, Königstuhl 17, 69117 Heidelberg, Germany e-mail: robitaille@mpia.de

2 Department of Astronomy, Yale University, PO Box 208101, New Haven, CT 06510, USA

3 Hubble Fellow

${ }^{4}$ Space Telescope Science Institute, 3700 San Martin Drive, Baltimore, MD 21218, USA

5 Harvard-Smithsonian Center for Astrophysics, 60 Garden Street, Cambridge, MA 02138, USA

6 Center for Astrophysics and Space Astronomy, University of Colorado, Boulder, CO 80309, USA

7 Department of Astronomy, Columbia University, Pupin Hall, 550W 120th St., New York, NY 10027, USA

8 Department of Astronomy and Astrophysics, University of Toronto, 50 Saint George Street, Toronto, ON M5S3H4, Canada

9 Argonne National Laboratory, High Energy Physics Division, 9700 South Cass Avenue, Argonne, IL 60439, USA

10 Department of Astronomy, Ohio State University, Columbus, OH 43210, USA

11 S.V.National Institute of Technology, 395007 Surat., India

12 Independent developer

13 Max-Planck-Institut für Kernphysik, PO Box 103980, 69029 Heidelberg, Germany

14 European Southern Observatory, Karl-Schwarzschild-Str. 2, 85748 Garching bei München, Germany

15 Laboratoire d'Astrophysique de Marseille, OAMP, Université Aix-Marseille et CNRS, 13388 Marseille, France

16 ETH Zürich, Institute for Astronomy, Wolfgang-Pauli-Strasse 27, Building HIT, Floor J, 8093 Zurich, Switzerland

17 Gemini Observatory, Casilla 603, La Serena, Chile

18 LIGO Laboratory, California Institute of Technology, 1200 E. California Blvd., Pasadena, CA 91125, USA

19 Center for Cosmology and Particle Physics, New York University, New York, NY 10003, USA

20 Department of Physics and Astronomy, Louisiana State University, Nicholson Hall, Baton Rouge, LA 70803, USA

21 Research School of Astronomy and Astrophysics, Australian National University, Mount Stromlo Observatory, via Cotter Road, Weston Creek ACT 2611, Australia

${ }^{22}$ SAAO, PO Box 9, Observatory 7935, 7925 Cape Town, South Africa

23 Joint Astronomy Centre, 660 N. A'ohōkū Place, Hilo, HI 96720, USA

24 Department of Astronomy, Cornell University, Ithaca, NY 14853, USA

${ }^{25}$ Gemini Observatory, 670 N. A'ohōkū Place, Hilo, HI 96720, USA

26 Oxford Astrophysics, Denys Wilkinson Building, Keble Road, Oxford OX1 3RH, UK

27 Department of Physics and Astronomy, University College London, London WC1E 6BT, UK

28 NASA Goddard Space Flight Center, X-ray Astrophysics Lab Code 662, Greenbelt, MD 20771, USA

${ }^{29}$ Laboratoire AIM, CEA Saclay, Bât. 709, 91191 Gif-sur-Yvette, France

${ }^{30}$ Leibniz-Institut für Astrophysik Potsdam (AIP), An der Sternwarte 16, 14482 Potsdam, Germany

Received 12 June 2013 / Accepted 23 July 2013

\section{ABSTRACT}

We present the first public version (v0.2) of the open-source and community-developed Python package, Astropy. This package provides core astronomy-related functionality to the community, including support for domain-specific file formats such as flexible image transport system (FITS) files, Virtual Observatory (VO) tables, and common ASCII table formats, unit and physical quantity conversions, physical constants specific to astronomy, celestial coordinate and time transformations, world coordinate system (WCS) support, generalized containers for representing gridded as well as tabular data, and a framework for cosmological transformations and conversions. Significant functionality is under active development, such as a model fitting framework, VO client and server tools, and aperture and point spread function (PSF) photometry tools. The core development team is actively making additions and enhancements to the current code base, and we encourage anyone interested to participate in the development of future Astropy versions.

Key words. methods: data analysis - methods: miscellaneous - virtual observatory tools 


\section{Introduction}

The Python programming language ${ }^{1}$ has become one of the fastest-growing programming languages in the astronomy community in the last decade (see e.g. Greenfield 2011, for a recent review). While there have been a number of efforts to develop Python packages for astronomy-specific functionality, these efforts have been fragmented, and several dozens of packages have been developed across the community with little or no coordination. This has led to duplication and a lack of homogeneity across packages, making it difficult for users to install all the required packages needed in an astronomer's toolkit. Because a number of these packages depend on individual or small groups of developers, packages are sometimes no longer maintained, or simply become unavailable, which is detrimental to long-term research and reproducibility.

Motivated by these issues, the Astropy project was started in 2011 out of a desire to bring together developers across the field of astronomy in order to coordinate the development of a common set of Python tools for astronomers and simplify the landscape of available packages. The project has grown rapidly, and to date, over 200 individuals are signed up to the development mailing list for the Astropy project ${ }^{2}$.

One of the primary aims of the Astropy project is to develop a core astropy package that covers much of the astronomyspecific functionality needed by researchers, complementing more general scientific packages such as NumPy (Oliphant 2006; Van Der Walt et al. 2011) and SciPy (Jones et al. 2001), which are invaluable for numerical array-based calculations and more general scientific algorithms (e.g. interpolation, integration, clustering). In addition, the Astropy project includes work on more specialized Python packages (which we call affiliated packages) that are not included in the core package for various reasons: for some the functionality is in early stages of development and is not robust; the license is not compatible with Astropy; the package includes large files; or the functionality is mature, but too domain-specific to be included in the core package.

The driving interface design philosophy behind the core package is that code using astropy should result in concise and easily readable code, even by those new to Python. Typical operations should appear in code similar to how they would appear if expressed in spoken or written language. Such an interface results in code that is less likely to contain errors and is easily understood, enabling astronomers to focus more of their effort on their science objectives rather than interpreting obscure function or variable names or otherwise spending time trying to understand the interface.

In this paper, we present the first public release (v0.2) of the astropy package. We provide an overview of the current capabilities (Sect. 2), our development workflow (Sect. 3), and planned functionality (Sect. 4). This paper is not intended to provide a detailed documentation for the package (which is available online ${ }^{3}$ ), but is rather intended to give an overview of the functionality and design.

\section{Capabilities}

This section provides a broad overview of the capabilities of the different astropy sub-packages, which covers units

\footnotetext{
1 http://www.python.org

2 https://groups.google.com/forum/?fromgroups\#! forum/ astropy-dev

3 http://docs.astropy.org
}

and unit conversions (Sect. 2.1), absolute dates and times (Sect. 2.2), celestial coordinates (Sect. 2.3), tabular and gridded data (Sect. 2.4), common astronomical file formats (Sect. 2.5), world coordinate system (WCS) transformations (Sect. 2.6), and cosmological utilities (Sect. 2.7). We have illustrated each section with simple and concise code examples, but for more details and examples, we refer the reader to the online documentation ${ }^{3}$.

\subsection{Units, quantities, and physical constants}

The astropy .units sub-package provides support for physical units. It originates from code in the pynbody package (Pontzen et al. 2013), but has been significantly enhanced in behavior and implementation (with the intent that pynbody will eventually become interoperable with astropy .units). This sub-package can be used to attach units to scalars and arrays, convert from one set of units to another, define custom units, define equivalencies for units that are not strictly the same (such as wavelength and frequency), and decompose units into base units. Unit definitions are included in both the International System of Units (SI) and the Centimeter-Gram-Second (CGS) systems, as well as a number of astronomy- and astrophysics-specific units.

\subsubsection{Units}

The astropy. units sub-package defines a Unit class to represent base units, which can be manipulated without attaching them to values, for example to determine the conversion factor from one set of units to another. Users can also define their own units, either as standalone base units or by composing other units together. It is also possible to decompose units into their base units, or alternatively search for higher-level units that are identical.

This sub-package includes the concept of "equivalencies" in units, which is intended to be used where there exists an equation that provides a relationship between two different physical quantities. A standard astronomical example is the relationships between the frequency, wavelength and energy of a photon - it is common practice to treat such units as equivalent even though they are not strictly comparable. Such a conversion can be carried out in astropy.units by supplying an equivalency list (see Fig. 1). The inclusion of these equivalencies is an important improvement over existing unit-handling software, which typically does not have this functionality. Equivalencies are also included for monochromatic flux densities, which allows users to convert between $F_{v}$ and $F_{\lambda}$, and users can easily implement their own equivalencies.

There are multiple string representations for units used in the astronomy community. The flexible image transport system (FITS) standard (Pence et al. 2010) defines a unit standard, as well as both the Centre de Données astronomiques de Strasbourg (CDS; Ochsenbein 2000) and NASA/Goddard's Office of Guest Investigator Programs (OGIP; George \& Angelini 1995). In addition, the International Virtual Observatory Alliance (IVOA) has a forthcoming VOUnit standard (Derriere et al. 2012) in an attempt to resolve some of these differences. Rather than choose one of these, astropy.units supports most of these standards (OGIP support is planned for the next major release of astropy), and allows the user to select the appropriate one when reading and writing unit string definitions to and from external file formats. 
The Astropy Collaboration: Astropy

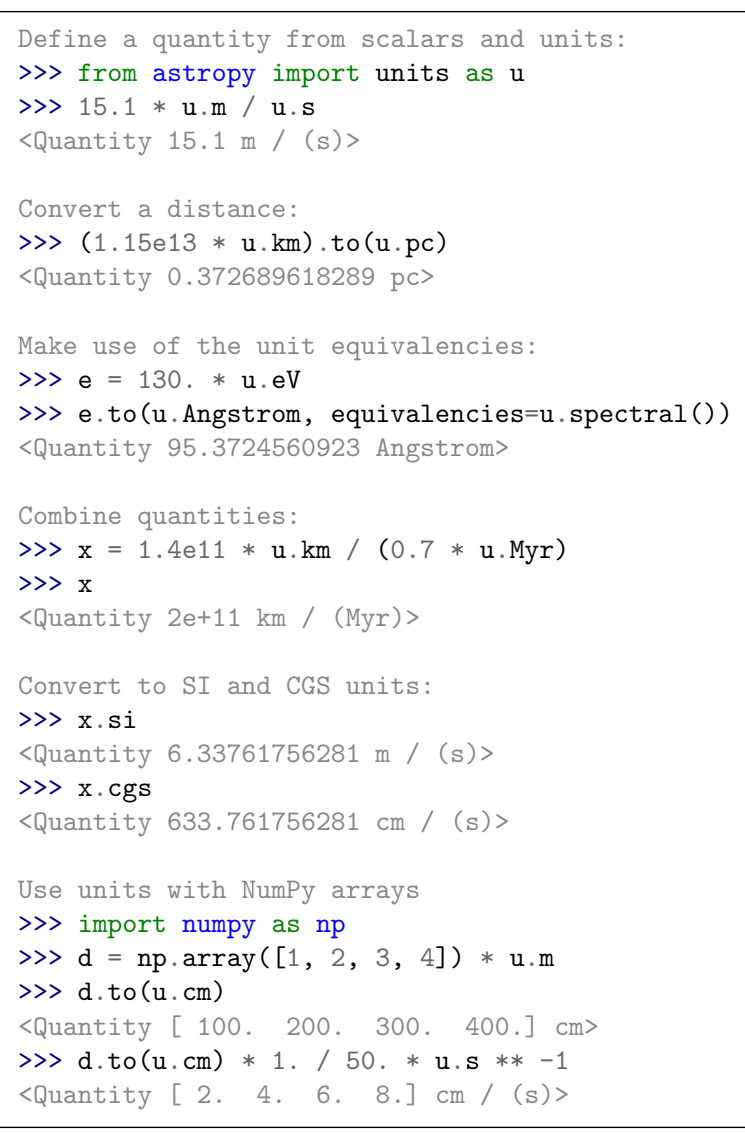

Fig. 1. Quantity conversion using the astropy . units sub-package.

\subsubsection{Quantities and physical constants}

While the previous section described the use of the astropy.units sub-package to manipulate the units themselves, a more common use-case is to attach the units to quantities, and use them together in expressions. The astropy. units package allows units to be attached to Python scalars, or NumPy arrays, producing Quantity objects. These objects support arithmetic with other numbers and Quantity objects while preserving their units. For multiplication and division, the resulting object will retain all units used in the expression. The final object can then be converted to a specified set of units or decomposed, effectively canceling and combining any equivalent units and returning a Quantity object in some set of base units. This is demonstrated in Fig. 1.

Using the .to() method, Quantity objects can easily be converted to different units. The units must either be dimensionally equivalent, or users should pass equivalencies through the equivalencies argument (cf. Sect. 2.1.1 or Fig. 1). Since Quantity objects can operate with NumPy arrays, it is very simple and efficient to convert the units on large datasets.

The Quantity objects are used to define a number of useful astronomical constants included in astropy. constants, each with an associated unit (where applicable) and additional metadata describing their provenance and uncertainties. These can be used along with Quantity objects to provide a convenient framework for computing any quantity in astronomy. Figure 2 includes a simple example that shows how the gravitational force between two bodies can be calculated in Newtons using physical constants and user-specified quantities.

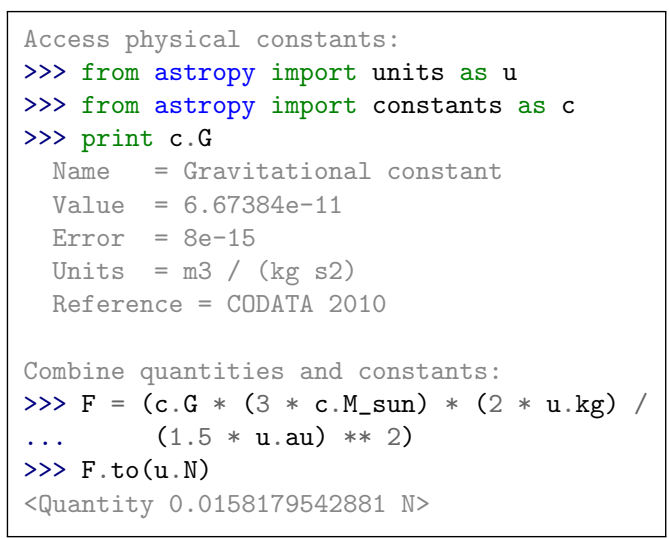

Fig. 2. Using the astropy. constants sub-package.

Table 1. Supported time scales for astropy.time

\begin{tabular}{ll}
\hline \hline Scale & Description \\
\hline TAI & International atomic time \\
TCB & Barycentric coordinate time \\
TCG & Geocentric coordinate time \\
TDB & Barycentric dynamical time \\
TT & Terrestrial time \\
UT1 & Universal time \\
UTC & Coordinated universal time \\
\hline
\end{tabular}

\subsection{Time}

The astropy.time package provides functionality for manipulating times and dates. Specific emphasis is placed on supporting time scales (e.g. UTC, TAI, UT1) and time formats or representations (e.g. JD, MJD, ISO 8601) that are used in astronomy (Guinot \& Seidelmann 1988; Kovalevsky 2001; Wallace 2011). Examples of using this sub-package are provided in Fig. 3.

The most common way to use astropy.time is to create a Time object by supplying one or more input time values as well as the time format or representation and time scale of those values. The input time(s) can either be a single scalar such as "2010-01-01 $00: 00: 00$ " or 2455348.5 or a sequence of such values; the format or representation specifies how to interpret the input values, such as ISO, JD, or Unix time; and the scale specifies the time standard used for the values, such as coordinated universal time (UTC), terrestial time (TT), or international atomic time (TAI). The full list of available time scales is given in Table 1. Many of these formats and scales are used within astronomy, and it is especially important to treat the different time scales properly when converting between celestial coordinate systems. To facilitate this, the Time class makes the conversion to a different format such as Julian Date straightforward, as well as the conversion to a different time scale, for instance from UTC to TT. We note that the Time class includes support for leap seconds in the UTC time scale.

This package is based on a derived version of the Standards of Fundamental Astronomy (SOFA) time and calendar library ${ }^{4}$ (Wallace 2011). Leveraging the robust and well-tested SOFA routines ensures that the fundamental time scale conversions are being computed correctly. An important feature of the SOFA time library which is supported by astropy.time is that each time is represented as a pair of double-precision (64-bit) floating-point values, which enables extremely high precision

4 http://www.iausofa.org 


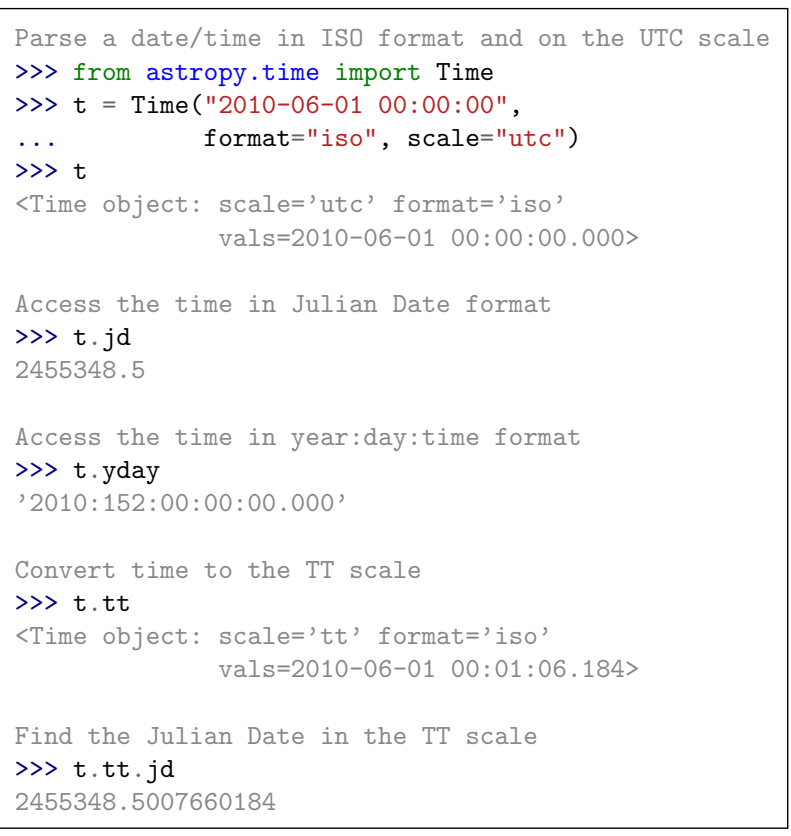

Fig. 3. Time representation and conversion using the astropy.time sub-package.

time computations. Using two 64-bit floating-point values allows users to represent times with a dynamic range of 30 orders of magnitude, providing for example times accurate to better than a nanosecond over timescales of tens of Gyr. All time scale conversions are done by vectorized versions of the SOFA routines using Cython (Behnel et al. 2011), a Python package that makes it easy to use $\mathrm{C}$ code in Python.

\subsection{Celestial coordinates}

An essential element of any astronomy workflow is the manipulation, parsing, and conversion of astronomical coordinates. This functionality is provided in Astropy by the astropy.coordinates sub-package. The aim of this package is to provide a common application programming interface (API) for Python astronomy packages that use coordinates, and to relieve users from having to (re)implement extremely common utilities. To achieve this, it combines API and implementation ideas from existing Python coordinates packages. Some aspects, such as coordinate transformation approaches from kapteyn (Terlouw \& Vogelaar 2012) and class structures resembling astropysics (Tollerud 2012), have already been implemented. Others, such as the frames of palpy (Jenness \& Berry 2013) and pyast (Berry \& Jenness 2012) or the ephemeris system of pyephem (Rhodes 2011), are still under design for astropy. By combining the best aspects of these other packages, as well as testing against them, astropy.coordinates seeks to provide a high-quality, flexible Python coordinates library.

The sub-package has been designed to present a natural Python interface for representing coordinates in computations, simplify input and output formatting, and allow straightforward transformation between coordinate systems. It also supports implementation of new or custom coordinate systems that work consistently with the built-in systems. A future design goal is to seamlessly support arbitrarily large data sets.

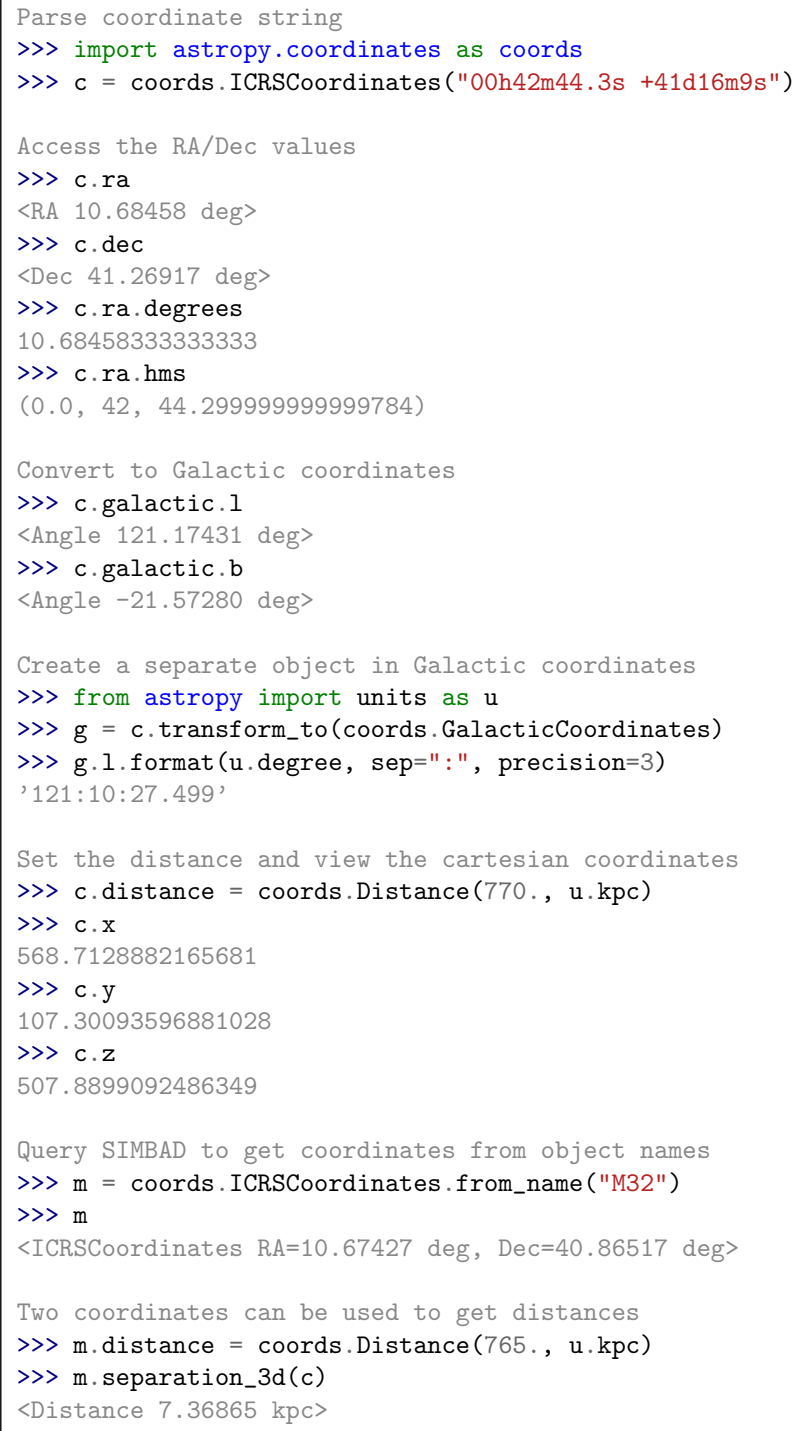

Fig. 4. Celestial coordinate representation and conversion.

Figure 4 shows some typical usage examples for astropy.coordinates. Coordinate objects are created using standard Python object instantiation via a Python class named after the coordinate system (e.g., ICRSCoordinates). Astronomical coordinates may be expressed in a myriad of ways: the classes support string, numeric, and tuple value specification through a sophisticated input parser. A design goal of the input parser is to be able to determine the angle value and unit from the input alone if a person can unambiguously determine them. For example, an astronomer seeing the input string $12 \mathrm{~h} 53 \mathrm{~m} 11.5123 \mathrm{~s}$ would understand the units to be in hours, minutes, and seconds, so this value is alone sufficient to pass to the angle initializer. This functionality is built around the Angle object, which can be instantiated and used on its own. It provides additional functionality such as string formatting and mechanisms to specify the valid bounds of an angle. As a convenience, it is also possible to query the online SIMBAD database to resolve the name of a source (see Fig. 4 for an example showing how to find the ICRS coordinates of M 32).

5 http://simbad.u-strasbg.fr 
The coordinate classes represent different coordinate systems, and provide most of the user-facing functionality for astropy. coordinates. The systems provide customized initializers and appropriate formatting and representation defaults. For some classes, they also contain added functionality specific to a subset of systems, such as code to precess a coordinate to a new equinox. The implemented systems include a variety of equatorial coordinate systems (ICRS, FK4, and FK5), Galactic coordinates, and horizontal (Alt/Az) coordinates, and modern (IAU 2006/200A) precession/nutation models for the relevant systems. Coordinate objects can easily be transformed from one coordinate system to another: Fig. 4 illustrates the most basic use of this functionality to convert a position on the sky from ICRS to Galactic coordinates. Transformations are provided between all coordinate systems built into version v0.2 of Astropy, with the exception of conversions from celestial to horizontal coordinates. Future versions of Astropy will include additional common systems, including ecliptic systems, supergalactic coordinates, and all necessary intermediate coordinate systems for the IAU 2000/2006 equatorial-to-horizontal mapping (e.g., Soffel et al. 2003; Kaplan 2005).

A final significant feature of astropy.coordinates is support for line-of-sight distances. While the term "celestial coordinates" can be taken to refer to only on-sky angles, in astropy.coordinates a coordinate object is conceptually treated as a point in three dimensional space. Users have the option of specifying a line of sight distance to the object from the origin of the coordinate system (typically the origin is the Earth or solar system barycenter). These distances can be given in physical units or as redshifts. The astropy.coordinates sub-package will in the latter case transparently make use of the cosmological calculations in astropy. cosmology (cf. Sect. 2.7) for conversion to physical distances. Figure 4 illustrates an application of this information in the form of computing three-dimensional distances between two objects.

The astropy.coordinates sub-package was designed such that it should be easy for a user to add new coordinate systems. This flexibility is achieved in astropy. coordinates through the internal use of a transformation graph, which keeps track of a network of coordinate systems and the transformations between them. When a coordinate object is to be transformed from one system into another, the package determines the shortest path on the transformation graph to the new system and applies the necessary sequence of transformations. Thus, implementing a new coordinate system simply requires implementing one pair of transformations to and from a system that is already connected to the transformation graph. Once this pair is specified, astropy.coordinates can transform from that coordinate system to any other in the graph. An example of a user-defined system is provided in the documentation ${ }^{6}$, illustrating the definition of a coordinate system useful for a specific scientific task.

\subsection{Tables and Gridded data}

Tables and $n$-dimensional data arrays are the most common forms of data encountered in astronomy. The Python community has various solutions for tables, such as NumPy structured arrays or DataFrame objects in Pandas (McKinney 2012) to name only a couple. For $n$-dimensional data the NumPy ndarray is the most popular.

\footnotetext{
6 http://docs.astropy.org/en/vQ.2.4/coordinates/ sgr-example.html
}

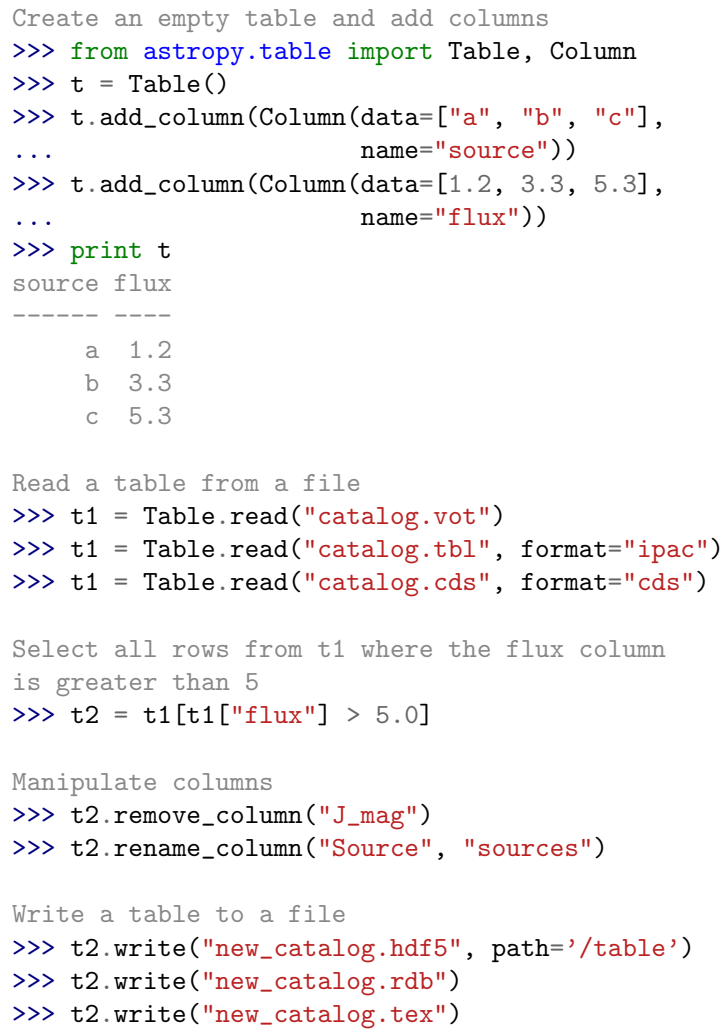

Fig. 5. Table input/output and manipulation using the astropy.table sub-package.

However, for use in astronomy all of these implementations lack some key features. The data that is stored in arrays and tables often contains vital metadata: the data is associated with units, and might also contain additional arrays that either mask or provide additional attributes to each cell. Furthermore, the data often includes a set of keyword-value pairs and comments (such as FITS headers). Finally, the data comes in a plethora of astronomy specific formats (FITS, specially formatted ASCII tables, etc.), which are not recognized by the pre-existing packages.

The astropy.table and astropy.nddata sub-packages contain classes (Table and NDData) that try to alleviate these problems. They allow users to represent astronomical data in the form of tables or $n$-dimensional gridded datasets, including all metadata. Examples of usage of astropy. table are shown in Fig. 5.

The Table class provides a high-level wrapper to NumPy structured arrays, which are essentially arrays that have fields (or columns) with heterogeneous data types, and any number of rows. NumPy structured arrays are however difficult to modify, so the Table class makes it easy for users to create a table from columns, add and remove columns or rows, and mask values from the table. Furthermore, tables can be easily read from and written to common file formats using the Table. read and Table.write methods. These methods are connected to sub-packages in astropy.io such as astropy.io.ascii (Sect. 2.5.2) and astropy.io.votable (Sect. 2.5.3), which allow ASCII and VO tables to be seamlessly read or written respectively.

In addition to providing easy manipulation and input or output of table objects, the Table class allows units to be 
specified for each column using the astropy . units framework (Sect. 2.1), and also allows the Table object to contain arbitrary metadata (stored in Table.meta).

Similarly, the NDData class provides a way to store $n$-dimensional array data easily and builds upon the NumPy ndarray class. The actual data is stored in an ndarray, which allows for easy compatibility with other scientific packages. In addition to keyword-value metadata, the NDData class can store a boolean mask with the same dimensions as the data, several sets of flags ( $n$-dimensional arrays that store attributes for each cell of the data array), uncertainties, units, and a transformation between array-index coordinate system and other coordinate systems (cf. Sect. 2.6). In addition, the NDData class intends to provide methods to arithmetically combine the data in a meaningful way. NDData is not meant for direct user interaction but more for providing a framework for higher-level subclasses that can represent for example spectra or astronomical images.

\subsection{File formats}

\subsubsection{FITS}

Support for reading and writing FITS files is provided by the astropy.io.fits sub-package, which at the time of writing is a direct port of the PyFITS ${ }^{7}$ project (Barrett \& Bridgman 1999). Users already familiar with PyFITS will therefore feel at home with this package.

The astropy.io.fits sub-package implements all features from the FITS standard (Pence et al. 2010) such as images, binary tables, and ASCII tables, and includes common compression algorithms. Header-data units (HDUs) are represented by Python classes, with the data itself stored using NumPy arrays, and with the headers stored using a Header class. Files can easily be read and written, and once in memory can be easily modified. This includes support for transparently reading from and writing to gzip-compressed FITS files as well as files using the tiled image compression standard. Figure 6 shows a simple example of how to open an existing FITS file, access and modify the header and data, and write a new file back to disk.

Creating new FITS files is also made simple. Since the code in this sub-package has been developed over more than a decade, it has been made to work with an extensive variety of FITS files, including ones that deviate from the FITS standard. This includes support for deprecated formats such as GROUPS HDUs as well as more obscure non-standard HDU types such as FITS HDUs which allow encapsulating multiple FITS files within FITS files. Support is also included for common but nonstandard header conventions such as CONTINUE cards and ESO HIERARCH cards. Two command-line utilities for working with FITS files are packaged with Astropy: fitscheck can be used to validate FITS files against the standard. fitsdiff can be used to compare two FITS files on a number of criteria, and also includes a powerful API for programmatically comparing FITS files.

Because the interface is exactly the same as that of PyFITS, users may directly replace PyFITS with Astropy in existing code by changing import statements such as import pyfits to from astropy.io import fits as pyfits without any additional code changes. Although PyFITS will continue to be released as a separate package in the near term, the long term plan is to discontinue PyFITS releases in favor of Astropy. It is expected that direct support of PyFITS will end mid-2014, so

\footnotetext{
7 http://www.stsci.edu/institute/software_hardware/
}

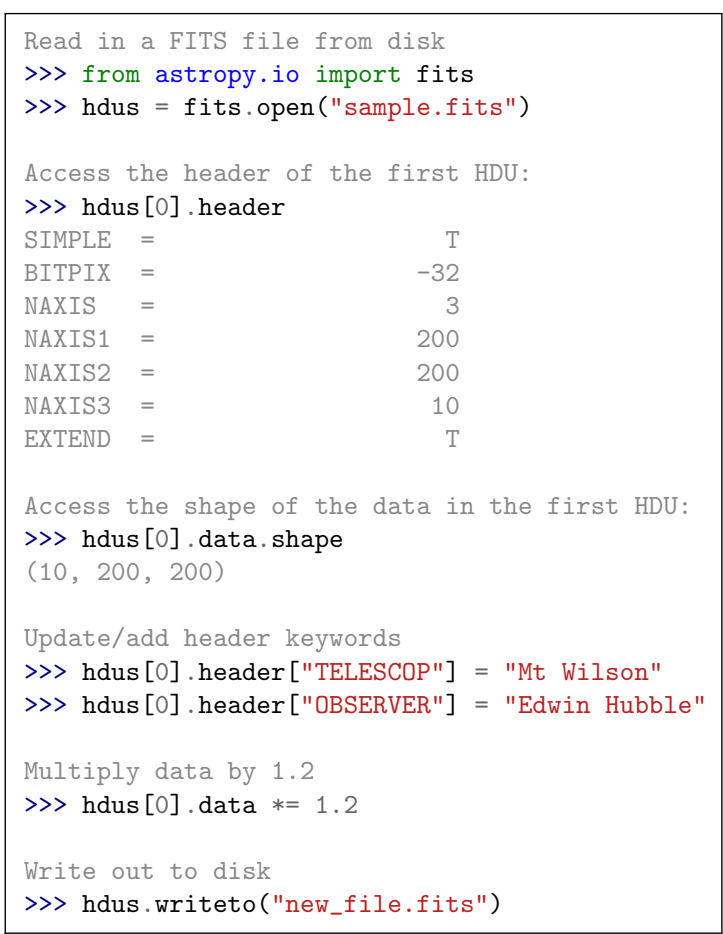

Fig. 6. Accessing data in FITS format.

users of PyFITS should plan to make suitable changes to support the eventual transition to Astropy.

Becoming integrated with Astropy as the astropy.io. fits sub-package will greatly enhance future development on the existing PyFITS code base in several areas. First and perhaps foremost is integration with Astropy's Table interface (Sect. 2.4) which is much more flexible and powerful than PyFITS' current table interface. We will also be able to integrate Astropy's unit support (Sect. 2.1) in order to attach units to FITS table columns as well as header values that specify units in their comments in accordance with the FITS standard. Finally, as the PyWCS package has also been integrated into Astropy as astropy.wcs (Sect. 2.6), tighter association between data from FITS files and their WCS will be possible.

\subsubsection{ASCII table formats}

The astropy .io.ascii sub-package (formerly the standalone project asciitable $e^{8}$ ) provides the ability to read and write tabular data for a wide variety of ASCII-based formats. In addition to generic formats such as space-delimited, tab-delimited or comma-separated values, astropy.io.ascii provides classes for specialized table formats such as $\mathrm{CDS}^{9}$, IPAC ${ }^{10}$, IRAF DAOphot (Stetson 1987), and LaTeX. Also included is a flexible class for handling a wide variety of fixed-width table formats. Finally, this sub-package is designed to be extensible, making it easy for users to define their own readers and writers for any other ASCII formats.

\footnotetext{
8 https://asciitable.readthedocs.org

9 http://vizier.u-strasbg.fr/doc/catstd.htx

${ }^{10}$ http://irsa.ipac.caltech.edu/applications/DDGEN/
} 


\subsubsection{Virtual Observatory (VO) tables}

The astropy.io.votable sub-package (formerly the standalone project vo.table) provides full support for reading and writing VOTable format files versions 1.1, 1.2, and the proposed 1.3 (Ochsenbein et al. 2004, 2009). It efficiently stores the tables in memory as NumPy structured arrays. The file is read using streaming to avoid reading in the entire file at once and greatly reducing the memory footprint. VOTable files compressed using the gzip and bzip2 algorithms are supported transparently, as are VOTable files where the table data is stored in an external FITS file.

It is possible to convert any one of the tables in a VOTable file to a Table object (Sect. 2.4), where it can be edited and then written back to a VOTable file without any loss of data.

The VOTable standard is not strictly adhered to by all VOTable file writers in the wild. Therefore, astropy.io.votable provides a number of tricks and workarounds to support as many VOTable sources as possible, whenever the result would not be ambiguous. A validation tool (volint) is also provided that outputs recommendations to improve the standard compliance of a given file, as well as validate it against the official VOTable schema.

\subsection{World coordinate systems}

The astropy.wcs sub-package contains utilities for managing WCS transformations in FITS files. These transformations map the pixel locations in an image to their real-world units, such as their position on the celestial sphere. This library is specific to WCS as it relates to FITS as described in the FITS WCS papers (Greisen \& Calabretta 2002; Calabretta \& Greisen 2002; Greisen et al. 2006) and is distinct from a planned Astropy package that will handle WCS transformations in general, regardless of their representation.

This sub-package is a wrapper around the wcslib library ${ }^{11}$. Since all of the FITS header parsing is done using wcslib, it is assured the same behavior as the many other tools that use wcslib. On top of the basic FITS WCS support, it adds support for the simple imaging polynomial (SIP) convention and table lookup distortions (Calabretta et al. 2004; Shupe et al. 2005). Each of these transformations can be used independently or together in a fixed pipeline. The astropy. wcs sub-package also serves as a useful FITS WCS validation tool, as it is able to report on many common mistakes or deviations from the standard in a given FITS file.

As mentioned above, the long-term plan is to build a "generalized" WCS for mapping world coordinates to image coordinates (and vice versa). While only in early planning stages, such a package would aim to not be tied to the FITS representation used for the current astropy.wcs. Such a package would also include closer connection to other parts of Astropy, for example astropy. coordinates (Sect. 2.3).

\subsection{Cosmology}

The astropy . cosmology sub-package contains classes for representing widely used cosmologies, and functions for calculating quantities that depend on a cosmological model. It also contains a framework for working with less frequently employed cosmologies that may not be flat, or have a time-varying pressure to density ratio, $w$, for dark energy. The quantities that can

\footnotetext{
11 http://www .atnf.csiro.au/people/mcalabre/WCS/
}

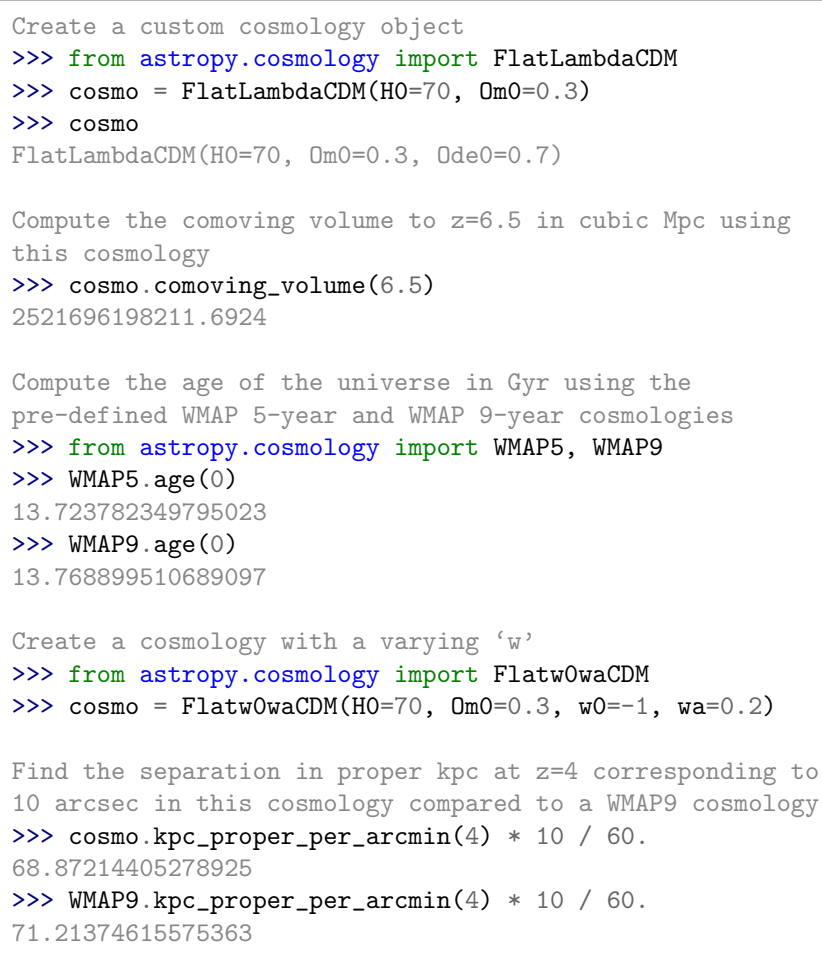

Fig. 7. Cosmology utilities.

be calculated are generally taken from those described by Hogg (1999). Some examples are the angular diameter distance, comoving distance, critical density, distance modulus, lookback time, luminosity distance, and Hubble parameter as a function of redshift.

The fundamental model for this sub-package is that any given cosmology is represented by a class. An instance of this class has attributes giving all the parameters required to specify the cosmology uniquely, such as the Hubble parameter, CMB temperature and the baryonic, cold dark matter, and dark energy densities at $z=0$. One can then use methods of this class to perform calculations using these parameters.

Figure 7 shows how the FlatLambdaCDM class can be used to create an object representing a flat $\Lambda \mathrm{CDM}$ cosmology, and how the methods of this object can be called to calculate the comoving volume, age and transverse separation at a given redshift. Further calculations can be performed using the many methods of the cosmology object as described in the Astropy documentation. For users who are more comfortable using a procedural coding style, these methods are also available as functions that take a cosmology class instance as a keyword argument.

The sub-package provides several pre-defined cosmology instances corresponding to commonly used cosmological parameter sets. Currently parameters from the WMAP 5-year (Komatsu et al. 2009), 7-year (Komatsu et al. 2011) and 9-year results (Hinshaw et al. 2012) are included (the WMAP5, WMAP7, and WMAP9 classes). The parameters from the Planck results (Planck Collaboration 2013) will be included in the next release of Astropy. There are several classes corresponding to non-flat cosmologies, and the most common dark energy models are supported: a cosmological constant, constant $w$, and $w(a)=w_{0}+$ $w_{a}(1-a)$ (e.g. Chevallier \& Polarski 2001; Linder 2003, here $a$ is the scale factor). Figure 7 gives examples showing how to use the pre-defined cosmologies, and how to define a new cosmology 
with a time-varying dark energy $w(a)$. Any other arbitrary cosmology can be represented by sub-classing one of the basic cosmology classes.

All of the code in the sub-package is tested against the webbased cosmology calculator by Wright (2006) and two other widely-used calculators $^{12,13}$. In cases when these calculators are not precise enough to enable a meaningful comparison, the code is tested against calculations performed with MatHEMATICA.

\section{Development approach}

A primary guiding philosophy of Astropy is that it is developed for and (at least in part) by the astronomy user community. This ensures the interface is designed with the workflow of working astronomers in mind. At the same time, it aims to make use of the expertise of software developers to design code that encourages good software practices such as a consistent and clean API, thorough documentation, and integrated testing. It is also dedicated to remaining open source to enable wide adoption and render input from all users easier, and is thus released with a 3-clause BSD-style license (a license of this sort is "permissive" in that it allows usage of the code for any purposes as long as notice of the Astropy copyright and disclaimers of warranty are given). Achieving these aims requires code collaboration between over 30 geographically-distributed developers, and here we describe our development workflow with the hope that it may be replicated by other astronomy software projects that are likely to have similar needs.

To enable this collaboration, we have made use of the GitHub $^{14}$ open source code hosting and development platform. The main repository for astropy is stored in a git ${ }^{15}$ repository on GitHub, and any non-trivial changes are made via pull requests, which are a mechanism for submitting code for review by other developers prior to merging into the main code base. This workflow aids in increasing the quality, documentation and testing of the code to be included in astropy. Not all contributions are necessarily accepted - community consensus is needed for incorporating major new functionality in astropy, and any new feature has to be justified to avoid implementing features that are only useful to a minority of users, but may cause issues in the future.

At the time of writing, astropy includes several thousand tests, which are small units of code that check that functions, methods, and classes in astropy are behaving as expected, both in terms of scientific correctness and from a programming interface perspective. We make use of continuous integration, which is the process of running all the tests under various configurations (such as different versions of Python or NumPy, and on different platforms) in order to ensure that the package is held to the highest standard of stability. In particular, any change made via a pull request is subject to extensive testing before being merged into the core repository. For the latter, we make use of Travis ${ }^{16}$, while for running more extensive tests across Linux, MacOS X, and Windows, we make use of Jenkins ${ }^{17}$ (both are examples of continuous integration systems).

This development workflow has worked very well so far, allowing contributions by many developers, and blurring the line

\footnotetext{
12 http://wwW . kempner .net/cosmic.php

13 http://www . icosmos.co.uk

14 http://www.github.com

15 http://git-scm. com

16 https://travis-ci.org

17 http://jenkins-ci.org
}

between developers and users. Indeed, users who encounter bugs and who know how to fix them can submit suggested changes. We have also implemented a feature that means that anyone reading the documentation at http://docs.astropy.org can suggest improvements to the documentation with just a few clicks in a web browser without any prior knowledge of the git version control system.

\section{Planned functionality}

Development on the Astropy package is very active, and in addition to some of the incremental improvements to existing subpackages described in the text, we are focusing on implementing major new functionality in several areas for the next (v0.3) release (some of which have already been implemented in the publicly-available developer version):

- improving interoperability between packages, which includes for example seamlessly integrating the astropy .units framework across all sub-packages;

- adding support for NumPy arrays in the coordinates subpackage, which will allow the efficient representation and conversions of coordinates in large datasets;

- supporting more file formats for reading and writing Table and NDData objects;

- implementing a VO cone search tool (Williams et al. 2011);

- implementing a generalized model-fitting framework;

- implementing statistical functions commonly used in Astronomy.

In the longer term, we are already planning the following major functionality:

- image analysis tools, including aperture and point spread function (PSF) photometry;

- spectroscopic analysis tools;

- generalized WCS transformations beyond the FITS WCS standard;

- a SAMP server/client (ported from the SAMPy ${ }^{18}$ package);

- support for the Simple Image Access Protocol (SIAP; Tody et al. 2011);

- support for the Table Access Protocol (TAP; Louys et al. 2011) is under consideration.

and undoubtedly the core functionality will grow beyond this. In fact, the astropy package will likely remain a continuouslyevolving package, and will thus never be considered "complete" in the traditional sense.

\section{Summary}

We have presented the first public release of the Astropy package (v0.2), a core Python package for astronomers. In this paper we have described the main functionality in this release, which includes:

- Units and unit conversions (Sect. 2.1).

- Absolute dates and times (Sect. 2.2).

- Celestial coordinate systems (Sect. 2.3).

- Tabular and gridded data (Sect. 2.4).

- Support for common astronomical file formats (Sect. 2.5).

- WCS transformations (Sect. 2.6).

- Cosmological calculations (Sect. 2.7).

18 http://pythonhosted.org/sampy 
We also briefly described our development approach (Sect. 3), which has enabled an international collaboration of scientists and software developers to create and contribute to the package. We outlined our plans for the future (Sect. 4) which includes more interoperability of sub-packages, as well as new functionality.

We invite members of the community to join the effort by adopting the Astropy package for their own projects, reporting any issues, and whenever possible, developing new functionality.

Acknowledgements. We thank the referee, Igor Chiligarian, for suggestions that helped improve this paper. We would like to thank the NumPy, SciPy (Jones et al. 2001), IPython and Matplolib communities for providing their packages which are invaluable to the development of Astropy. We thank the GitHub (http: //www.github.com) team for providing us with an excellent free development platform. We also are grateful to Read the Docs (https://readthedocs . org/), Shining Panda (https://www. shiningpanda-ci.com/), and Travis (https://www.travis-ci.org/) for providing free documentation hosting and testing respectively. Finally, we would like to thank all the astropy users that have provided feedback and submitted bug reports. The contribution by $\mathrm{T}$. Aldcroft and D. Burke was funded by NASA contract NAS8-39073. The name resolution functionality shown in Fig. 4 makes use of the SIMBAD database, operated at CDS, Strasbourg, France.

\section{References}

Barrett, P. E., \& Bridgman, W. T. 1999, in Astronomical Data Analysis Software and Systems VIII, ASP Conf. Ser., 172, 483

Behnel, S., Bradshaw, R., Citro, C., et al. 2011, Computing in Science Engineering, 13, 31

Berry, D. S., \& Jenness, T. 2012, in Astronomical Data Analysis Software and Systems XXI, eds. P. Ballester, D. Egret, \& N. P. F. Lorente, ASP Conf. Ser., 461,825

Calabretta, M. R., \& Greisen, E. W. 2002, A\&A, 1077

Calabretta, M. R., Valdes, F., Greisen, E. W., \& Allen, S. L. 2004, in Astronomical Data Analysis Software and Systems (ADASS) XIII, eds. F. Ochsenbein, M. G. Allen, \& D. Egret, ASP Conf. Ser., 314, 551

Chevallier, M., \& Polarski, D. 2001, Int. J. Mod. Phys. D, 10, 213

Derriere, S., Gray, N., Louys, M., et al. 2012, Units in the VO, Version 1.0, IVOA Proposed Recommendation 20 August $2012 \mathrm{ed}$.

George, I., \& Angelini, L. 1995, Specification of Physical Units within OGIP (Office of Guest Investigator Programs) FITS files

Greenfield, P. 2011, in Astronomical Data Analysis Software and Systems XX, eds. I. N. Evans, A. Accomazzi, D. J. Mink, \& A. H. Rots, ASP Conf. Ser., 442,425
Greisen, E. W., \& Calabretta, M. R. 2002, A\&A, 1061

Greisen, E. W., Calabretta, M. R., Valdes, F. G., \& Allen, S. L. 2006, A\&A, 747

Guinot, B., \& Seidelmann, P. K. 1988, A\&A, 194, 304

Hinshaw, G., Larson, D., Komatsu, E., et al. 2012 [arXiv: 1212 . 5226]

Hogg, D. W. 1999, ArXiv Astrophysics e-prints [arXiv: astro-ph/9905116] Jenness, T., \& Berry, D. S. 2013, in ADASS XXII, eds. D. Friedel, M. Freemon, \& R. Plante (San Francisco: ASP), ASP Conf Ser., TBD, in press

Jones, E., Oliphant, T., \& Peterson, P. 2001, http://www . scipy.org/

Kaplan, G. H. 2005, US Naval Observatory Circulars, 179

Komatsu, E., Dunkley, J., Nolta, M. R., et al. 2009, ApJS, 180, 330

Komatsu, E., Dunkley, J., Nolta, M. R., et al. 2011, ApJS, 192, 18

Kovalevsky, J. 2001, in Journées 2000 - systèmes de référence spatio-temporels. J2000, a fundamental epoch for origins of reference systems and astronomical models, ed. N. Capitaine, 218

Linder, E. V. 2003, Phys. Rev. Lett., 90, 091301

Louys, M. Bonnarel, F., Shade, D., et al. 2011 [arXiv: 1111.1758]

McKinney, W. 2012, Python for Data Analysis (O’Reilly Media, Incorporated)

Ochsenbein, F. 2000, Astronomical Catalogues and Tables Adopted Standards, Version 2.0

Ochsenbein, F., et al. 2004, VOTable Format Definition, Version 1.1, International Virtual Observatory Alliance (IVOA)

Ochsenbein, F., Davenhall, C., Durand, D., et al. 2009, VOTable Format Definition, Version 1.2, International Virtual Observatory Alliance (IVOA)

Oliphant, T. 2006, A Guide to NumPy, 1 (Trelgol Publishing USA)

Pence, W. D., Chiappetti, L., Page, C. G., Shaw, R. A., \& Stobie, E. 2010, A\&A, 524, A42

Planck Collaboration 2013, A\&A, submitted [arXiv: 1303.5076]

Pontzen, A., Roškar, R., Stinson, G. S., et al. 2013, Pynbody: Astrophysics Simulation Analysis for Python (Astrophysics Source Code Library, ascl:1305.002)

Rhodes, B. C. 2011, PyEphem: Astronomical Ephemeris for Python (Astrophysics Source Code Library, ascl:1112.014)

Shupe, D. L., Moshir, M., Li, J., et al. 2005, in Astronomical Data Analysis Software and Systems XIV, eds. P. Shopbell, M. Britton, \& R. Ebert, ASP Conf. Ser., 347, 491

Soffel, M., Klioner, S. A., Petit, G., et al. 2003, AJ, 126, 2687

Stetson, P. B. 1987, PASP, 99, 191

Terlouw, J. P., \& Vogelaar, M. G. R. 2012, Kapteyn Package, version 2.2, Kapteyn Astronomical Institute, Groningen

Tody, D., Plante, R., \& Harrison, P. 2011 [arXiv: 1110. 0499]

Tollerud, E. 2012, Astropysics: Astrophysics utilities for python (Astrophysics Source Code Library, ascl:1207.007)

Van Der Walt, S., Colbert, S., \& Varoquaux, G. 2011, Computing in Science \& Engineering, 13, 22

Wallace, P. T. 2011, Metrologia, 48, 200

Williams, R., Hanisch, R., Szalay, A., \& Plante, R. 2011 [arXiv: 1110.0498]

Wright, E. L. 2006, PASP, 118, 1711 